\title{
SUPPLEMENTARY MATERIAL: ON A GENERAL DEFINITION OF DEPTH FOR FUNCTIONAL DATA
}

\author{
IRÈNE GIJBELS ${ }^{1}$ AND STANISLAV NAGY ${ }^{1,2}$
}

\begin{abstract}
This document contains the proofs of all new theoretical results stated in the main paper, detailed references to the results known from the literature, and several examples demonstrating the properties of the studied functional depths.
\end{abstract}

\section{S.1. Proof of Lemma 1}

The "if"-part of the statement is trivial. For the other implication, without loss of generality, assume that $X$ is symmetric around $\mathfrak{F} \ni \theta \equiv 0$, and set $Y=-X$. For $\mathfrak{F}=\mathbb{L}_{2}$, central symmetry of $X$ implies that $\langle X, u\rangle=\langle Y, u\rangle$ in distribution for each $u \in \mathbb{L}_{2}$. By Cuesta-Albertos et al. (2006, Proposition 2.1) this means that $X=Y$ in distribution. For $\mathfrak{F}=\mathcal{C}$, note that the dual of $\mathbb{L}_{2}$ can be canonically embedded into $\mathcal{C}^{*}$. Thus, if $X$ is centrally symmetric in $\mathcal{C}$, it is also centrally symmetric in $\mathbb{L}_{2}$, and by the first part of the proof, $X=Y$ in distribution in $\mathbb{L}_{2}$. Because each function in $\mathcal{C}$ is also in $\mathbb{L}_{2}$, this means that $X=Y$ in distribution also in $\mathcal{C}$.

In the rest of this document we provide the non-trivial proofs of the new results summarized in Table 1 of the main paper. We structure the material per statistical depth functional, and thus by column in Table 1 .

\section{S.2. $h$-DEPTH}

For $\mathrm{P}-2 \mathrm{C}$ and $\mathrm{P}-2 \mathrm{H}$, consider for $\mathfrak{F}=\mathbb{R}$ the uniform distribution on the set $[-2,-1] \cup$ $[1,2]$. This distribution is (halfspace, and centrally) symmetric in $\mathbb{R}$ about 0 . Taking $h=1$ in the definition of $h$-depth, we see that $D(0, P)=\int_{1}^{2} K_{h}(x) \mathrm{d} x \approx 0.136$. On the other hand, it can be evaluated that $D(1, P)=\int_{0}^{1} K_{h}(x) / 2 \mathrm{~d} x+\int_{2}^{3} K_{h}(x) / 2 \mathrm{~d} x \approx 0.181$, and $D(0, P)<\sup _{x \in \mathfrak{F}} D(x, P)$. P-3D was already shown not to be true in Section 2.3 of the main paper, see the right panel in Figure 1. P-3V follows easily from the fact that the kernel $K_{h}$ in $D_{h}$ decreases to zero, and the tightness of $P$, see Dudley (2002, Section 11.5). Verification of P-4 is straightforward - in fact, just as in Nagy (2015) it is possible to show that the set of functionals $\left\{K_{h}\left(\|x-\cdot\|_{\mathbb{L}_{2}}\right): \mathbb{L}_{2} \rightarrow \mathbb{R}: x \in \mathbb{L}_{2}\right\}$ is uniformly bounded and equicontinuous, and P-6 with P-6U can then be concluded by Dudley (2002, Corollary 11.3.4).

\footnotetext{
1 KU Leuven, Department of Mathematics and Leuven Statistics Research Centre (LStAT), Belgium

2 Charles University, Department of Probability and Math. Statistics, Czech REPUBLIC

E-mail addresses: irene.gijbels@kuleuven.be, nagy@karlin.mff.cuni.cz.

Date: August 2, 2017.
} 


\section{S.3. RANDOM TUKEY DEPTH}

By the very definition of $D_{R T}$ it is evident that its theoretical properties relate closely to those of the original Tukey depth $D_{T}$. For the latter, the equivalents of all the positive results for $D_{R T}$ from Table 1 can be shown in $\mathbb{R}^{p}$ for $D_{T}$ (Donoho and Gasko, 1992, Zuo and Serfling, 2000). P-0 is easy to establish. P-1S follows from ZS-1 for $D_{T}$.

Denote by $\mathbb{U}$ the (finite-dimensional, thus closed) linear subspace of $\mathbb{L}_{2}$ spanned by the elements of $\mathfrak{U}$. Also, write $\mathbb{U}^{\perp}$ for its orthogonal complement in $\mathbb{L}_{2}$ (Dudley, 2002, Section 5.3). By Dudley (2002, Theorem 5.3.8), each $x \in \mathbb{L}_{2}$ can be uniquely decomposed into $y \in \mathbb{U}$ and $z \in \mathbb{U}^{\perp}$ so that $x=y+z$. This way, also $X \sim P_{X}$ can be written as $Y+Z$, where $Y$ is a random variable in $\mathbb{U}$ with distribution $P_{Y}$, and $Z$ a random variable in $\mathbb{U}^{\perp}$ with distribution $P_{Z}$. Now, we can write $D_{R T}\left(x, P_{X}\right)=D_{T}\left(y, P_{Y}\right)$ for any $x$ and $X$. Analogously, considering $x_{1}, x_{2} \in \mathbb{L}_{2}$ and $\alpha \in[0,1]$, we can write $D_{R T}\left(x_{1}+\alpha\left(x_{1}-\right.\right.$ $\left.\left.x_{2}\right), P_{X}\right)=D_{T}\left(y_{1}+\alpha\left(y_{1}-y_{2}\right), P_{Y}\right)$, and P-3D is true for $D_{R T}$ because ZS-3 is true for $D_{T}$. Condition $\mathrm{P}-3 \mathrm{~V}$ is not valid for $D_{R T}$, even though for $D_{T}$ it is. This can be seen by taking a sequence $x_{n} \in \mathbb{L}_{2}, n=1,2, \ldots$ such that each corresponding $y_{n}$ is equal to $y \in \mathbb{U}$, but $\left\|x_{n}\right\|_{\mathbb{L}_{2}} \rightarrow \infty$ and $D_{T}\left(y, P_{Y}\right)>0$, which can be done in the infinite-dimensional space $\mathbb{L}_{2}$. Then we have $\lim _{n \rightarrow \infty} D_{R T}\left(x_{n}, P_{X}\right)=\lim _{n \rightarrow \infty} D_{T}\left(y_{n}, P_{Y}\right)>0$. Using the same argument, it is easy to see that neither $\mathrm{P}-2 \mathrm{C}$ nor $\mathrm{P}-2 \mathrm{H}$ can be true, as $D_{R T}$ assigns the maximal depth to any point which projects into the maximizer of $D_{T}$ for $Y$. P-4 is easy to see to be valid for $D_{R T}$. P-6U is true since it is true for $D_{T}$ (see Nagy et al., 2016b, Theorem 14), but only if $P_{Y}$ is absolutely continuous, or for empirical measures.

\section{S.4. BAND DEPTH}

P-0, P-2C, P-2H and P-3D are violated by $D_{J}$ (Chakraborty and Chaudhuri, 2014a, Theorem 4). P-1S, P-1F, P-3V and P-4 are shown in López-Pintado and Romo (2009, Theorem 3). P-6U is shown to fail in general (over compact sets, or for the sequence of empirical measures) in Gijbels and Nagy (2015). To extend the last result, we now demonstrate that P-6 may be violated even for $X \sim P$ such that the distribution of each $X(v)$ is absolutely continuous. Let $U$ and $V$ be two independent, real-valued random variables, distributed uniformly over $[0,1]$. Define $X \sim P$ by $\mathrm{P}(X \equiv-U)=1 / 2$ and $\mathrm{P}(X(v)=|V-v|$ for $v \in \mathcal{V})=1 / 2$, with $\mathcal{V}=[0,1]$. It is easy to see that $X$ is supported in a compact subset of $\mathcal{C}$, and that $X(v)$ is absolutely continuous for all $v \in \mathcal{V}$. For $\nu=1,2, \ldots$ define $X_{\nu} \sim P_{\nu}$ by $X_{\nu} \equiv X$ if $X(0)<0$, and $X_{\nu} \equiv X-1 / \nu$ otherwise. Evidently, $X_{\nu}$ converges in distribution to $X$. Yet, for $x \equiv 0$ (and $J=2$, for instance), we have $D_{J}(x, P)=1 / 2$, but $D_{J}\left(x, P_{\nu}\right)=0$ for any $\nu=1,2 \ldots$, and P-6 is not valid.

\section{S.5. MODIFIED BAND DEPTH}

The theoretical properties of this depth, as a representative of the integrated depths for functional data, are examined thoroughly in Nagy et al. (2016b). The proofs of all the results related to $D_{M J}$ from Table 1 can be found in Appendix A.3 of that paper.

\section{S.6. HALFREGION DEPTH}

P-0, P-2C, P-2H and P-3D are violated by $D_{H R}$ as demonstrated by Chakraborty and Chaudhuri (2014a, Theorem 4), see also Kuelbs and Zinn $(2013,2015)$. The verification of P-1S and P-1F is done in López-Pintado and Romo (2011). P-3V and P-4 are true for $D_{H R}$ by López-Pintado and Romo (2011, Propositions 3 and 6). P-6U is shown to fail 
in general (over compact sets, or for the sequence of empirical measures) in Gijbels and Nagy (2015). To see that P-6 may fail also for $X$ with absolutely continuous marginal distributions, one can use the same counterexample as for the band depth.

\section{S.7. MODIFIED HALFREGION DEPTH}

Verification of P-0, P-1S, P-1F and P-4 is straightforward, see also López-Pintado and Romo (2011). By Nagy et al. (2016b, Theorem 22), for $P$ such that all its marginals are absolutely continuous, P-6U is satisfied. Moreover, the uniform consistency of $D_{M H R}$ is true for any $P$ by Theorem 21 therein. Finally, to see that $\mathrm{P}-2 \mathrm{C}$ and $\mathrm{P}-2 \mathrm{H}$ are not true for $D_{M H R}$, take $P_{X} \in \mathcal{P}$ for $\mathcal{V}=[0,1]$ given by $\mathrm{P}(X(v)=1-2 v)=1 / 2$, $\mathrm{P}(X(v)=2 v-1)=1 / 2 . P$ is (halfspace, and centrally) symmetric around the function $\theta \equiv 0$. However, $D_{M H R}(\theta, P)=1 / 2$, while for $x(v)=1-2 v$ we have $D_{M H R}(x, P)=$ $D_{M H R}(-x, P)=3 / 4$. Also, this example shows that P-3D is violated too for $D_{M H R}$, by noting that $\theta \equiv 1 / 2 x+(1-1 / 2)(-x)$.

\section{S.8. TUKEY DEPTH}

For $D_{T}$, condition P-0 is violated by Dutta et al. (2011, Section 4). For conditions P-1-P-6, the results follow from the corresponding properties of the depth $D_{T}$ defined in finite-dimensional spaces, see for instance Nagy et al. (2016b, Appendix A.1).

To see that P-6 and P-6U cannot be true (for empirical measures over compact sets), consider $W$ the usual Wiener process on $\mathcal{V}=[0,1]$, and define a random function $X \sim P$ by setting

$$
X(v)= \begin{cases}-1 & \text { if } W(v) \leq-1 \\ W(v) & \text { if }-1<W(v)<1 \\ 1 & \text { if } W(v) \geq 1\end{cases}
$$

For any $u \in \mathfrak{F}$, the distribution of $\langle X, u\rangle$ is symmetric around 0 , and for $x \equiv 0$ we have $D_{T}(x, P)=1 / 2$. For almost surely any random sample $X_{1}, \ldots, X_{n}$ from $P$, however, there exists $u \in \mathfrak{F}$ such that $\langle x, u\rangle<\min _{i=1, \ldots, n}\left\langle X_{i}, u\right\rangle$, using the same argument as in Gijbels and Nagy (2016, Example 5). Thus, $D_{T}\left(x, P_{n}\right)=0$ almost surely for all $n$, and the depth is not consistent, even though $P$ is supported in a compact set, and $P_{n}$ is taken as the sequence of empirical measures.

\section{S.9. Spatial DEPTH}

P-0, P-1, P-1S, P-4, P-6 and P-6U are true by Chakraborty and Chaudhuri (2014b, Section 4).

Now we verify $\mathrm{P}-2 \mathrm{C}$. Without loss of generality, assume that $X \sim P$ is centrally symmetric around $\mathfrak{F} \ni \theta \equiv 0$, i.e. for $\varphi \in \mathfrak{F}^{*}, \varphi(X)$ is centrally symmetric around 0 . Because

$$
\left|\mathbb{E} \varphi\left(\frac{X}{\|X\|_{\mathbb{L}_{2}}}\right)\right| \leq \sup _{x \in \mathfrak{F}:\|x\|_{\mathbb{L}_{2}} \leq 1} \varphi(x)<\infty \quad \text { for all } \varphi \in \mathfrak{F}^{*},
$$

this implies

$$
\mathbb{E} \varphi\left(\frac{X-\theta}{\|X-\theta\|_{\mathbb{L}_{2}}}\right)=\mathbb{E} \frac{1}{\|X\|_{\mathbb{L}_{2}}} \varphi(X)=0=\varphi(0) \quad \text { for all } \varphi \in \mathfrak{F}^{*} .
$$


Therefore, the Pettis integral of $(X-\theta) /\|X-\theta\|_{\mathbb{L}_{2}}$ is the zero function. Because the Bochner integral coincides with the Pettis integral if they both exist (Fremlin and Mendoza, 1994),

$$
\mathbb{E} \frac{X-\theta}{\|X-\theta\|_{\mathbb{L}_{2}}}=0
$$

and $D_{S}(\theta, P)=1$.

To see that $\mathrm{P}-2 \mathrm{H}$ may fail to hold, take $\mathcal{V}=[0,1]$, and consider $P$ an equal priors mixture of $P_{1}$, the Dirac measure at $\mathfrak{F} \ni \theta \equiv 0$, and $X_{2} \sim P_{2}$ for $X_{2} \equiv U$ such that $U$ is an exponentially distributed real-valued random variable with mean 1 . Then $P$ is halfspace symmetric around $\theta$. On the other hand,

$$
D_{S}(\theta, P)=1-\left\|\mathbb{E} \frac{X-\theta}{\|X-\theta\|_{\mathbb{L}_{2}}}\right\|_{\mathbb{L}_{2}}=1-\left\|\frac{1}{2} \mathbb{E} \frac{0}{0}+\frac{1}{2} \mathbb{E} \frac{X_{2}}{\left\|X_{2}\right\|_{\mathbb{L}_{2}}}\right\|_{\mathbb{L}_{2}}=\frac{1}{2},
$$

but for $x \equiv u$ for $u>0$

$$
D_{S}(x, P)=1-\left\|\mathbb{E} \frac{X-x}{\|X-x\|_{\mathbb{L}_{2}}}\right\|_{\mathbb{L}_{2}}=\exp (-u),
$$

and $D_{S}(x, P)>D_{S}(\theta, P)$ for $x \equiv u>0$ small.

Condition P-3V follows from the tightness of $P$ in separable Banach spaces (see Dudley, 2002, Section 11.5). In finite-dimensional spaces $\mathbb{R}^{p}$ with $p>1$, there exist distributions $Q$ such that an analogue of the spatial depth $D_{S}$ in $\mathbb{R}^{p}$ (Chaudhuri, 1996) violates ZS-3 for $Q$, see Nagy (2017). For the functional version of this result, consider $p=2$, take $Q$ as above in $\mathbb{R}^{2}$, and let $b_{1}, b_{2} \in \mathfrak{F}$ be orthonormal in $\mathfrak{F}$. Define $X \sim P \in \mathcal{P}$ by $X=\sum_{j=1}^{2} q_{j} b_{j}$, where $\left(q_{1}, q_{2}\right) \sim Q$. Then, it is easy to show that for $x=\sum_{j=1}^{2} x_{j} b_{j}$

$$
\left\|\mathbb{E} \frac{X-x}{\|X-x\|_{\mathbb{L}_{2}}}\right\|_{\mathbb{L}_{2}}=\left\|\mathbb{E} \frac{\left(q_{1}, q_{2}\right)-\left(x_{1}, x_{2}\right)}{\left\|\left(q_{1}, q_{2}\right)-\left(x_{1}, x_{2}\right)\right\|}\right\|,
$$

where on the right hand side the Euclidean norm in $\mathbb{R}^{2}$ is used. Thus, $D_{S}(x, P)$ equals the spatial depth in $\mathbb{R}^{2}$ of the coefficients of $x$ w.r.t. the coefficients of $X$ (in the basis $\left.\left(b_{1}, b_{2}\right)\right)$, and P-3D is violated for $D_{S}$ in functional spaces, just as ZS-3 is in $\mathbb{R}^{2}$.

For atomic distributions, the depth $D_{S}$ violates $\mathrm{P}-4$ already in $\mathbb{R}$.

\section{S.10. $L^{\infty}$ DEPTH}

P-3D, P-3V, and P-4 are verified by Long and Huang (2016). P-0 is violated for any $X \sim P$ with $\mathbb{E}\|X\|_{\infty}=\infty$. For any $x \in \mathfrak{F}, P \in \mathcal{P}$ and $f(x)=a x$ for $a>0$, we have $D_{L^{\infty}}\left(f(x), P_{f(X)}\right)=D_{L^{\infty}}(x, P) /\left(a+(1-a) D_{L^{\infty}}(x, P)\right)$, i.e. P-1, and P-1S are satisfied only if $a=1$. Condition $\mathrm{P}-2 \mathrm{G}$ is true because for any centred (finite-dimensional) Gaussian random vector $U$ the function $u \mapsto \mathbb{E}\|u-U\|_{\infty}$ is known to be minimized at $u=0$. The functional analogue follows immediately. To see that P-2C and P-2H are not valid, consider the following example. Let $X \sim P \in \mathcal{P}$ for $\mathcal{V}=[0,1]$ be such that $X(0)$, is distributed uniformly on the set $[-1,-1 / 2] \cup[1 / 2,1]$, and set

$$
X(v)= \begin{cases}X(0)(1-2 v) & \text { for } v \in[0,1 / 2] \\ 0 & \text { for } v \in(1 / 2,1] .\end{cases}
$$

Distribution $P$ is centrally, and halfspace symmetric around $\theta \equiv 0$, and

$$
D_{L^{\infty}}(\theta, P)=\left(1+\mathbb{E}\|X-\theta\|_{\infty}\right)^{-1}=(1+\mathbb{E}|X(0)|)^{-1}=4 / 7 .
$$


Take $x \in \mathfrak{F}$ defined by

$$
x(v)= \begin{cases}0 & \text { for } v \in[0,1 / 2], \\ v / 2-1 / 4 & \text { for } v \in(1 / 2,1] .\end{cases}
$$

Then, $x$ is not a centre of symmetry for $P$, yet still

$$
D_{L^{\infty}}(x, P)=\left(1+\mathbb{E}\|X-x\|_{\infty}\right)^{-1}=(1+\mathbb{E} \max \{|X(0)|, 1 / 4\})^{-1}=4 / 7 .
$$

To see that P-6 is not satisfied, consider first $\left\{U_{\nu}\right\}_{\nu=1}^{\infty}$ a sequence of positive real random variables such that $U_{\nu}$ converges in distribution to a positive real random variable $U$, all $\mathbb{E} U_{\nu}$ and $\mathbb{E} U$ exist, but at the same time $\mathbb{E} U_{\nu}$ does not converge to $\mathbb{E} U$. Such a sequence can be constructed, for instance, using the technique from Nagy et al. (2016a, Example 1). Now, define $X_{\nu} \sim P_{\nu}$ to be a constant function such that $X_{\nu} \equiv U_{\nu}$, and set $X \equiv U$. The sequence $\left\{X_{\nu}\right\}_{\nu=1}^{\infty}$ converges in distribution to $X \sim P$. For $x \equiv 0$, however, $D_{L^{\infty}}\left(x, P_{\nu}\right)=1 /\left(1+\mathbb{E} U_{\nu}\right) \not \rightarrow D_{L^{\infty}}(x, P)=1 /(1+\mathbb{E} U)$, and P-6 is not valid, even though all $\mathbb{E}\left\|X_{\nu}\right\|_{\infty}=\mathbb{E} U_{\nu}<\infty$, and $\mathbb{E}\|X\|_{\infty}=\mathbb{E} U<\infty$.

Finally, we verify that P-6U is true for $D_{L^{\infty}}$ over compact subsets $C \subset \mathfrak{F}$, for $P_{n}$, $n=1,2, \ldots$, the empirical measures from $P$. For this, note first that because for $f(t)=$ $1 /(1+t)$ we have $|f(s)-f(t)| \leq|s-t|$ for $s, t \in[0, \infty)$, we can bound

$$
\sup _{x \in C}\left|D_{L^{\infty}}\left(x, P_{n}\right)-D_{L^{\infty}}(x, P)\right| \leq \sup _{x \in C}\left|\frac{1}{n} \sum_{i=1}^{n}\left\|x-X_{i}\right\|_{\infty}-\mathbb{E}\|x-X\|_{\infty}\right|,
$$

where $X_{1}, \ldots, X_{n}$ are the random sample functions from $P$ corresponding to $P_{n}$. On the right hand side we have an empirical process, indexed by the set of mappings

$$
g_{x}: \mathfrak{F} \rightarrow \mathbb{R}: z \mapsto\|x-z\|_{\infty}, \quad \text { for } x \in C .
$$

This set is Lipschitz in its parameter, as for any $x, y \in C, z \in \mathfrak{F}$, by the reverse triangle inequality,

$$
\left|g_{x}(z)-g_{y}(z)\right| \leq\|x-y\|_{\infty} .
$$

Van der Vaart and Wellner (1996, Theorem 2.7.11) give that the bracketing numbers of the class (S.1) are bounded by the covering numbers of $C$, which are finite as $C$ is compact. Thus, we can conclude that P-6U is true as stated (Van der Vaart and Wellner, 1996, Theorem 2.4.1).

\section{S.11. INFIMAL DEPTH}

Conditions P-1S, P-1F, P-3V and P-4 were verified in Mosler (2013, Section 2.4). P-0, P-2G, P-2H and P-3D are violated by the same argument as for the band depth. P-6 and P-6U were discussed in Gijbels and Nagy (2016, Section 5).

\section{REFERENCES}

Chakraborty, A. and Chaudhuri, P. (2014a). On data depth in infinite dimensional spaces. Ann. Inst. Statist. Math., 66(2):303-324.

Chakraborty, A. and Chaudhuri, P. (2014b). The spatial distribution in infinite dimensional spaces and related quantiles and depths. Ann. Statist., 42(3):1203-1231.

Chaudhuri, P. (1996). On a geometric notion of quantiles for multivariate data. J. Amer. Statist. Assoc., 91(434):862-872. 
Cuesta-Albertos, J. A., Fraiman, R., and Ransford, T. (2006). Random projections and goodness-of-fit tests in infinite-dimensional spaces. Bull. Braz. Math. Soc. (N.S.), $37(4): 477-501$.

Donoho, D. L. and Gasko, M. (1992). Breakdown properties of location estimates based on halfspace depth and projected outlyingness. Ann. Statist., 20(4):1803-1827.

Dudley, R. M. (2002). Real analysis and probability, volume 74 of Cambridge Studies in Advanced Mathematics. Cambridge University Press, Cambridge. Revised reprint of the 1989 original.

Dutta, S., Ghosh, A. K., and Chaudhuri, P. (2011). Some intriguing properties of Tukey's half-space depth. Bernoulli, 17(4):1420-1434.

Fremlin, D. H. and Mendoza, J. (1994). On the integration of vector-valued functions. Illinois J. Math., 38(1):127-147.

Gijbels, I. and Nagy, S. (2015). Consistency of non-integrated depths for functional data. J. Multivariate Anal., 140:259 - 282.

Gijbels, I. and Nagy, S. (2016). On smoothness of Tukey depth contours. Statistics, 50(5):1075-1085.

Kuelbs, J. and Zinn, J. (2013). Concerns with functional depth. ALEA Lat. Am. J. Probab. Math. Stat., 10(2):831-855.

Kuelbs, J. and Zinn, J. (2015). Half-region depth for stochastic processes. J. Multivariate Anal., 142:86-105.

Long, J. P. and Huang, J. Z. (2016). A study of functional depths. arXiv preprint arXiv:1506.01332.

López-Pintado, S. and Romo, J. (2009). On the concept of depth for functional data. J. Amer. Statist. Assoc., 104(486):718-734.

López-Pintado, S. and Romo, J. (2011). A half-region depth for functional data. Comput. Statist. Data Anal., 55(4):1679-1695.

Mosler, K. (2013). Depth statistics. In Becker, C., Fried, R., and Kuhnt, S., editors, Robustness and complex data structures, pages 17-34. Springer, Heidelberg.

Nagy, S. (2015). Consistency of h-mode depth. J. Stat. Plan. Inference, 165:91 - 103.

Nagy, S. (2017). Monotonicity properties of spatial depth. Statist. Probab. Lett., 129:373378.

Nagy, S., Gijbels, I., and Hlubinka, D. (2016a). Weak convergence of discretely observed functional data with applications. J. Multivariate Anal., 146:46 - 62. Special Issue on Statistical Models and Methods for High or Infinite Dimensional Spaces.

Nagy, S., Gijbels, I., Omelka, M., and Hlubinka, D. (2016b). Integrated depth for functional data: statistical properties and consistency. ESAIM Probab. Stat., 20:95-130.

Van der Vaart, A. W. and Wellner, J. A. (1996). Weak convergence and empirical processes. Springer Series in Statistics. Springer-Verlag, New York.

Zuo, Y. and Serfling, R. (2000). General notions of statistical depth function. Ann. Statist., 28(2):461-482. 